\title{
ASYMPTOTIC EXPANSION OF THE DISPERSION EQUATION OF LAMB WAVES IN PERIODICALLY LAYERED ELASTIC MEDIA
}

\author{
Bui Thanh Tu${ }^{1}$, Pham Chi Vinh ${ }^{1}$, Nguyen Thi Khanh Linh ${ }^{2}$ \\ ${ }^{1}$ Hanoi University of Science \\ ${ }^{2}$ Publishing House for Science and Technology
}

\begin{abstract}
The present paper deals with the problem on Lamb waves propagation in periodically layered, compressible elastic media with initial deformations, in the case of long wavelength approximation (i.e. $\varepsilon=k . h<<1$, where $k$ is the wave number, $h$ is the thickness of one periodicity cell). With the assumption that $\varepsilon<<1$, the dispersion equation is written as:

$$
\frac{\omega^{2}}{k^{2}}=\Omega_{1}+\varepsilon \Omega_{2}+\varepsilon^{2} \Omega_{3}+\cdots=\sum_{m=0}^{\infty} \varepsilon^{m} \Omega_{m+1} .
$$

The main aim of this paper is to find formulae for determining the coefficients $\Omega_{i},(i \geq 1)$. In particular, we prove that $\Omega_{2 n}=0(n \geq 1)$, derive formulae for $\Omega_{1}, \Omega_{3}$, and construct recurrent formulae for $\Omega_{2 n+1}(n \geq 2)$. Based on these formulae, the solution with any order of accuracy can be obtained. This research is an extension of the investigation by Norris and Santosa [Norris A. and Santosa F., Wave Motion 16 (1992), 33-55] from SH waves (one-component waves) to Lamb waves (two-component waves).

Keyword. Lamb waves, Wave propagation, Periodically layered media, Asymptotic expansion.
\end{abstract}

\section{INTRODUCTION}

Wave propagation in elastic media has received an intensive consideration because of its wide range of applications in various fields in science such as seismology, acoustics, material sciences, construction,... (see for example, [1], [2]). Since thin structures are often encountered in engineering and technology, the wave propagation in thin layers, or longwave propagation, has been the subject of many investigations, see for instance [3]-[17] and references therein.

In the wave propagation studies, it is important to derive dispersion equations being of the form: $\omega=\omega\left(k, p_{1}, p_{2}, \ldots\right)$, where $\omega$ is the wave frequency, $k$ is the wave number, and $p_{i}(\mathrm{i}=1,2, \ldots)$ are the characteristic parameters of the material. The dispersion equations can be used to solve the direct (forward) problems which study the effects of material parameters on the wave velocity $c=\omega / k$ or to solve the inverse problems which determine material parameters from the measured values of the wave speed $c$. 
When the layers are thin, or the considered wave is a long wave (low-frequency wave), we have:

$$
0<\varepsilon=k . h<<1
$$

where $h$ is a typical length (it is the thickness of one periodicity cell for the periodically layered structures, for instance). Taking assumption (1) into account, the dispersion equation can be expanded into a power series of the small parameter $\epsilon$ as follows:

$$
c^{2}=\frac{\omega^{2}}{k^{2}}=\Omega_{1}+\varepsilon \Omega_{2}+\varepsilon^{2} \Omega_{3}+\ldots=\sum_{m=0}^{\infty} \Omega_{m+1} \varepsilon^{m}
$$

here the coefficients $\Omega_{m}$ need to be determined.

Norris and Santosa [6] studied the propagation of SH-wave (one-component wave) in a periodically layered infinite elastic medium without initial deformations. They derived the formulae of $\Omega_{1}, \Omega_{3}$ and proved that $\Omega_{2}=0$.

In this paper, the results obtained by Norris and Santosa [6] are extended to Lamb wave (two-component wave). In particular, we derive the expressions for $\Omega_{1}, \Omega_{3}$ and demonstrate $\Omega_{2}=0$. Moreover, we establish recurrent formulae for $\Omega_{2 n+1}(n \geq 2)$ and prove that $\Omega_{2 n}=0, \forall n \geq 1$. The material is assumed with initial deformations.

The layout of the paper is as follows. The problem setting and mathematical formulations are introduced in Section 2. The asymptotic analysis, the expressions for $\Omega_{1}, \Omega_{3}$ and the vanishing of $\Omega_{2}$ are discussed in Section 3. Section 4 presents recurrent formulae for calculating the higher-order coefficients $\Omega_{m}$ in the expansion (2).

\section{PROBLEM SETTING AND MATHEMATICAL FORMULATIONS}

Let us consider a periodically layered, infinite elastic medium. Each periodicity cell consists of $N$ different layers $(N \geq 2)$. Suppose that the material layers are compressible isotropic and subject to homogeneous initial deformations (see [18], [19]). At the initial state, we introduce a system of orthogonal Cartesian coordinates $O z_{1} z_{2} z_{3}$ in which the coordinate plane $O z_{1} z_{2}$ coincides with the bottom plane of the first material layer of the periodicity cell (see Fig. 1). By $h_{i}$ and $h$ we denote, respectively, the thickness of the $i$-th layer $(i=1, \ldots, N)$ and the thickness of one periodicity cell at the initial state, then we have:

$$
h=h_{1}+h_{2}+\cdots+h_{N} .
$$

Let us consider a Lamb wave propagating along the layers, in the $O z_{1}$-direction, and its displacement vector lies on the plane $O z_{1} z_{3}$. The components of the wave vector have the form $\vec{k}\left(k_{1}, k_{2}, k_{3}\right)=(k, 0,0)$, and non-zero components of the displacement vector of the Lamb wave are: $u_{1}=u_{1}\left(z_{1}, z_{3}, \tau\right), u_{3}=u_{3}\left(z_{1}, z_{3}, \tau\right)$, where $\tau$ is the time.

The following system of equations is used to study the problem (see [18], [19]):

$$
L_{m \alpha} u_{\alpha}=0 \quad m, \alpha=1,3,
$$

where: 


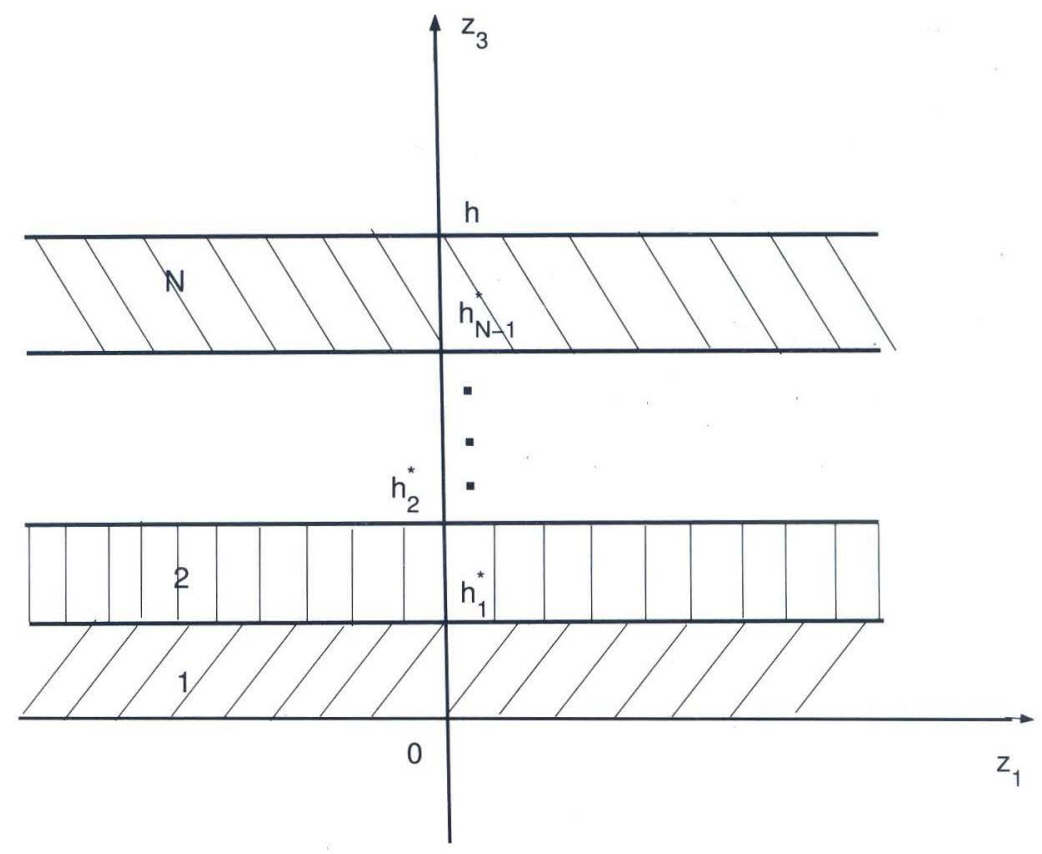

Fig. 1. The periodicity cell

$$
L_{m \alpha}=\frac{\partial}{\partial z_{i}}\left[\tilde{\omega}_{i m \alpha \beta}\left(z_{3}\right) \frac{\partial}{\partial z_{\beta}}\right]-\tilde{\rho}\left(z_{3}\right) \delta_{m \alpha} \frac{\partial^{2}}{\partial \tau^{2}} \quad, \quad i, \beta=1,3,
$$

$\tilde{\rho}\left(z_{3}\right)$ is the mass density of the medium at the initial state. Note that $\tilde{\omega}_{i m \alpha \beta}\left(z_{3}\right), \tilde{\rho}\left(z_{3}\right)$ are periodic functions of period $h$. They are constant on each interval $\left(h_{i-1}^{*}, h_{i}^{*}\right), i=1, \ldots, N$ (see [19]), where:

$$
h_{i}^{*}=h_{1}+h_{2}+\ldots+h_{i}, i=1,2, \ldots, N, h_{0}^{*}=0 .
$$

The surface tractions per unit area $\tilde{P}_{m}(m=1,3)$. at the initial state at the plane $z_{3}=$ const are calculated as follows (see [19]):

$$
\tilde{P}_{m}=\tilde{\omega}_{3 m \alpha \beta}\left(z_{3}\right) \cdot \frac{\partial u_{\alpha}}{\partial z_{\beta}}, \quad m=1,3 .
$$

Equations (4) are written in the matrix form as

$$
L \mathbf{u}=\tilde{\rho} \frac{\partial^{2} \mathbf{u}}{\partial \tau^{2}}
$$

where:

$$
L \mathbf{u}=\frac{\partial}{\partial z_{h}}\left(A_{h k} \frac{\partial \mathbf{u}}{\partial z_{k}}\right)
$$




$$
\begin{array}{rlrl}
\mathbf{u} & =\left[\begin{array}{l}
u_{1} \\
u_{3}
\end{array}\right], & & \\
A_{11} & =\left[\begin{array}{cc}
\tilde{\omega}_{1111} & 0 \\
0 & \tilde{\omega}_{1331}
\end{array}\right], & & A_{33}=\left[\begin{array}{cc}
\tilde{\omega}_{3113} & 0 \\
0 & \tilde{\omega}_{3333}
\end{array}\right], \\
A_{13} & =\left[\begin{array}{cc}
0 & \tilde{\omega}_{3311} \\
\tilde{\omega}_{3131} & 0
\end{array}\right], & A_{31}=\left[\begin{array}{cc}
0 & \tilde{\omega}_{3131} \\
\tilde{\omega}_{3311} & 0
\end{array}\right] .
\end{array}
$$

It should be noted that when the medium is at the natural state, we have

$$
\tilde{\omega}_{1111}=\tilde{\omega}_{3333}=\lambda+2 \mu, \quad \tilde{\omega}_{1331}=\tilde{\omega}_{3113}=\tilde{\omega}_{3131}=\mu, \quad \tilde{\omega}_{3311}=\lambda .
$$

In the matrix form, formula (6) becomes

$$
\tilde{P}=A_{31} \frac{\partial \mathbf{u}}{\partial z_{1}}+A_{33} \frac{\partial \mathbf{u}}{\partial z_{3}},
$$

where:

$$
\tilde{P}=\left[\begin{array}{l}
\tilde{P}_{1} \\
\tilde{P}_{3}
\end{array}\right] .
$$

We find the solution of equation (7) in the form

$$
\mathbf{u}=\mathbf{U}\left(z_{3}\right) e^{i\left(k z_{1}-\omega \tau\right)}
$$

Substituting (12) into (7) yields:

$$
\frac{d}{d z_{3}}\left(A_{33} \frac{d \mathbf{U}}{d z_{3}}+i k A_{31} \mathbf{U}\right)+i k A_{13} \frac{d \mathbf{U}}{d z_{3}}+\left(\tilde{\rho} \omega^{2} E-k^{2} A_{11}\right) \mathbf{U}=0
$$

where

$$
E=\left[\begin{array}{ll}
1 & 0 \\
0 & 1
\end{array}\right], \quad \mathbf{U}\left(\mathbf{z}_{3}\right)=\left[\begin{array}{l}
U_{1}\left(z_{3}\right) \\
U_{3}\left(z_{3}\right)
\end{array}\right] .
$$

Substitution of equation (12) into equation (10) leads to

$$
\tilde{P}=P\left(z_{3}\right) e^{i\left(k z_{1}-\omega \tau\right)}, \quad P=A_{31} \frac{d \mathbf{U}}{d z_{1}}+A_{33} \frac{d \mathbf{U}}{d z_{3}},
$$

where

$$
P=\left[\begin{array}{l}
P_{1} \\
P_{3}
\end{array}\right]
$$

Equation (13) can be rewritten as

$$
\frac{d}{k d z_{3}}\left(A_{33} \frac{d \mathbf{U}}{k d z_{3}}+i A_{31} \mathbf{U}\right)+i A_{13} \frac{d \mathbf{U}}{k d z_{3}}+\left(\tilde{\rho} \frac{\omega^{2}}{k^{2}} E-A_{11}\right) \mathbf{U}=0 .
$$

Denote $Y_{1}=\mathbf{U}, Y_{2}=A_{33} \frac{d \mathbf{U}}{k d z_{3}}+i A_{31} \mathbf{U}$, then equation (15) yields

$$
\frac{d \boldsymbol{Y}}{k d z_{3}}=B \cdot \boldsymbol{Y}
$$


where

$$
\boldsymbol{Y}=\left[\begin{array}{l}
Y_{1} \\
Y_{2}
\end{array}\right], \quad B=\left[\begin{array}{cc}
-i A_{33}^{-1} A_{31} & A_{33}^{-1} \\
-\left(A_{13} A_{33}^{-1} A_{31}+\tilde{\rho} \frac{\omega^{2}}{k^{2}}-A_{11}\right) & -i A_{13} A_{33}^{-1}
\end{array}\right]
$$

Introducing the new variable $y_{3}=z_{3} / h$, then equation (16) is written as:

$$
\frac{d \boldsymbol{Y}}{d y_{3}}=\varepsilon B \cdot \boldsymbol{Y}
$$

where

$$
B=\left[\begin{array}{cccc}
0 & b_{12} & b_{13} & 0 \\
b_{21} & 0 & 0 & b_{24} \\
b_{31}^{\prime} & 0 & 0 & b_{34} \\
0 & b_{42}^{\prime} & b_{43} & 0
\end{array}\right]
$$

in which

$$
\begin{aligned}
& b_{12}=b_{43}=-i \frac{\tilde{\omega}_{3131}}{\tilde{\omega}_{1}}, \\
& b_{13}=\frac{1}{\tilde{\omega}_{1}}, \\
& b_{21}=b_{34}=-i \frac{\tilde{\omega}_{3311}}{\tilde{\omega}_{3}}, \\
& b_{24}=\frac{1}{\tilde{\omega}_{3}}, \\
& b_{31}^{\prime}=-\left(\frac{\tilde{\omega}_{3311}^{2}}{\tilde{\omega}_{3}}+\tilde{\rho} \frac{\omega^{2}}{k^{2}}-\tilde{\omega}_{1111}\right), \\
& b_{42}^{\prime}=-\left(\frac{\tilde{\omega}_{3131}^{2}}{\tilde{\omega}_{3}}+\tilde{\rho} \frac{\omega^{2}}{k^{2}}-\tilde{\omega}_{3131}\right), \\
& \tilde{\omega}_{1}=\tilde{\omega}_{3113}, \quad \tilde{\omega}_{3}=\tilde{\omega}_{3333} .
\end{aligned}
$$

Note that $B\left(y_{3}\right)$ is a periodic function of period 1. In summary, we need to solve equation (18) on the interval $[0,1]$, with assumption $\varepsilon=k . h<<1$. The periodicity of the medium leads to condition

$$
\mathbf{Y}(1)=\mathbf{Y}(0)
$$

\section{ASYMPTOTIC SOLUTION, EXPRESSIONS FOR $\Omega_{1}, \Omega_{3}$}

Suppose that the layers are all thin, or the Lamb wave is a long-wave (low-frequency wave) which leads to $0<\varepsilon<<1$. As mentioned above, when $\varepsilon<<1$, the dispersion equation of the Lamb wave can be written as

$$
\frac{\omega^{2}}{k^{2}}=\Omega_{1}+\varepsilon \Omega_{2}+\varepsilon^{2} \Omega_{3}+\cdots+\varepsilon^{j-1} \Omega_{j}+\cdots=\sum_{m=0}^{\infty} \varepsilon^{m} \Omega_{m+1} .
$$


Our task now is to determine the coefficients $\Omega_{1}, \Omega_{2}, \Omega_{3} \ldots$. Substituting equation (22) into formulas (19), (20), matrix $B$ can be expressed as follows

$$
B=B_{0}+\varepsilon B_{1}+\varepsilon^{2} B_{2}+\cdots+\varepsilon^{j} B_{j}+\cdots,
$$

where

$$
\begin{gathered}
B_{j}=\tilde{\rho} \Omega_{j+1} L, \quad \forall j=1,2,3 \ldots, \\
L=\left[\begin{array}{llll}
0 & 0 & 0 & 0 \\
0 & 0 & 0 & 0 \\
1 & 0 & 0 & 0 \\
0 & 1 & 0 & 0
\end{array}\right], B_{0}=\left[\begin{array}{cccc}
0 & b_{12} & b_{13} & 0 \\
b_{21} & 0 & 0 & b_{24} \\
b_{31} & 0 & 0 & b_{34} \\
0 & b_{42} & b_{43} & 0
\end{array}\right],
\end{gathered}
$$

and

$$
b_{31}=-\left(\frac{\tilde{\omega}_{3311}^{2}}{\tilde{\omega}_{3}}+\tilde{\rho} \Omega_{1}-\tilde{\omega}_{1111}\right), \quad b_{42}=-\left(\frac{\tilde{\omega}_{3131}^{2}}{\tilde{\omega}_{3}}+\tilde{\rho} \Omega_{1}-\tilde{\omega}_{3131}\right) .
$$

In order to solve the equation (18), we seek $Y$ in the form

$$
\boldsymbol{Y}=Y_{0}+\varepsilon^{1} Y_{1}+\varepsilon^{2} Y_{2}+\varepsilon^{3} Y_{3}+\cdots=\sum_{n=0}^{\infty} \varepsilon^{n} Y_{n}
$$

We introduce new matrices $R_{n}\left(y_{3}\right)=\left[r_{i j}^{n}\left(y_{3}\right)\right]_{4 \times 4}, T_{n}\left(y_{3}\right)=\left[t_{i j}^{n}\left(y_{3}\right)\right]_{4 \times 4}$ and $S_{n}=\left[s_{i j}^{n}\right]_{4 \times 4}$ defined as follows

$$
\begin{aligned}
& R_{n}\left(y_{3}\right)=\sum_{m=0}^{n} \int^{y_{3}} B_{n-m} R_{m-1} ; \quad S_{n}=R_{n}(1) ; \quad \forall n=0,1,2, \ldots ; \\
& T_{n}\left(y_{3}\right)=\sum_{m=1}^{n} \int^{y_{3}} B_{n-m} R_{m-1}, \quad \forall n=1,2, \ldots ; \quad T_{0}=\int^{y_{3}} B_{0},
\end{aligned}
$$

where

$$
\int^{y_{3}} \Gamma=\int_{0}^{y_{3}} \Gamma(x) d x ; \quad R_{-1}=\operatorname{diag}(1,1,1,1)=I .
$$

From the above definitions, $R_{n}\left(y_{3}\right)$ relates to $T_{n}\left(y_{3}\right)$ by the relation

$$
R_{n}\left(y_{3}\right)=T_{n}\left(y_{3}\right)+\int^{y_{3}} B_{n}, \quad \forall n \geq 1
$$

Lemma 1:

$$
Y_{j}\left(y_{3}\right)=R_{j-1}\left(y_{3}\right) Y(0), \quad \forall j=1,2, \ldots
$$


Proof: Taking into account (23) and (26), we have from (18)

$$
\sum_{j=0}^{\infty} \varepsilon^{j} \frac{d Y_{j}}{d y_{3}}=\sum_{i=0}^{\infty} \varepsilon^{i+1} B_{i} \sum_{k=0}^{\infty} \varepsilon^{k} Y_{k} .
$$

The coefficients of the same order of $\varepsilon$ on both sides in (32) must be the same. Therefore, we have the following differential equations

$$
\begin{aligned}
\frac{d Y_{0}}{d y_{3}} & =0, \\
\frac{d Y_{1}}{d y_{3}} & =B_{0} Y_{0}, \\
\frac{d Y_{2}}{d y_{3}} & =B_{1} Y_{0}+B_{0} Y_{1}, \\
\frac{d Y_{3}}{d y_{3}} & =B_{2} Y_{0}+B_{1} Y_{1}+B_{0} Y_{2}, \\
\cdots & \\
\frac{d Y_{j}}{d y_{3}} & =\sum_{s=0}^{j-1} B_{j-s-1} Y_{s} .
\end{aligned}
$$

Taking integration of equations (33)-(36) leads to

$$
\begin{aligned}
Y_{0} \text { const }=Y(0) ; \\
Y_{1}\left(y_{3}\right)=\left(\int^{y_{3}} B_{0}\right) Y(0)=R_{0} Y(0) ; \\
Y_{2}\left(y_{3}\right)=\left(\int^{y_{3}} B_{1}+\int^{y_{3}} B_{0} S_{0}\right) Y(0)=R_{1} Y(0) ; \\
Y_{3}\left(y_{3}\right)=\left(\int^{y_{3}} B_{2}+\int^{y_{3}} B_{1} S_{1}+\int^{y_{3}} B_{0} S_{2}\right) Y(0)=R_{2} Y(0) .
\end{aligned}
$$

Thus, the statement (31) holds true for $j=1,2,3$. Assume that the statement (31) holds true for any $j=1,2,3 \ldots n-1$. We will prove that it also holds true for $j=n$. From (37), we have:

$$
\frac{d Y_{n}}{d y_{3}}=\sum_{s=0}^{n-1} B_{n-s-1} Y_{s} .
$$

From the inductive assumption: $Y_{s}=R_{s-1} Y(0)$ and the above equation, we have

$$
\frac{d Y_{n}}{d y_{3}}=\sum_{s=0}^{n-1} B_{n-s-1} R_{s-1} Y(0)
$$

Integration of this equation gives: 


$$
Y_{n}=\left(\sum_{s=0}^{n-1} \int^{y_{3}} B_{n-s-1} R_{s-1}\right) Y(0) \quad \text { or } \quad Y_{n}=R_{n-1} Y(0)
$$

Thus, statement (31) holds true for $j=n$ and it is valid for $\forall j=1,2, \ldots$

Substitution of (31) into (26) leads to

$$
\begin{aligned}
Y\left(y_{3}\right) & =\left[I+\varepsilon^{1} R_{0}\left(y_{3}\right)+\varepsilon^{2} R_{1}\left(y_{3}\right)+\varepsilon^{3} R_{2}\left(y_{3}\right)+\cdots\right] Y(0) \\
& =\left[\sum_{n=0}^{\infty} \varepsilon^{n} R_{n-1}\left(y_{3}\right)\right] Y(0) .
\end{aligned}
$$

Taking the periodicity condition (21) into account and noting that $S_{n}=R_{n}(1)$, from (42) we have

$$
\boldsymbol{Y}(1)=\left[I+\varepsilon^{1} S_{0}+\varepsilon^{2} S_{1}+\varepsilon^{3} S_{2}+\cdots\right] Y(0)=\left[\sum_{n=0}^{\infty} \varepsilon^{n} S_{n-1}\right] Y(0)
$$

or

$$
\boldsymbol{Y}(0)=\left[\sum_{n=0}^{\infty} \varepsilon^{n} S_{n-1}\right] Y(0)
$$

Equation (43) leads to

$$
\operatorname{det}\left[\sum_{n=0}^{\infty} \varepsilon^{n} S_{n}\right]=\operatorname{det}\left[S_{0}+\varepsilon^{1} S_{1}+\varepsilon^{2} S_{2}+\cdots+\varepsilon^{j} S_{j}+\cdots\right]=0 .
$$

Equation (44) can be written as

$$
\operatorname{det}\left[\sum_{i=0}^{\infty} \varepsilon^{i} A^{i}, \sum_{j=0}^{\infty} \varepsilon^{j} B^{j}, \sum_{k=0}^{\infty} \varepsilon^{k} C^{k}, \sum_{h=0}^{\infty} \varepsilon^{h} D^{h}\right]=0
$$

where $A^{j}, B^{j}, C^{j}, D^{j}$ is the 1 st, 2 nd, 3 th and 4 th column of matrix $S_{j}, j=0,1,2, \ldots$ Expansion of the first, second, third and forth column of the above determinant yields

$$
\sum_{i=0}^{\infty} \sum_{j=0}^{\infty} \sum_{k=0}^{\infty} \sum_{h=0}^{\infty} \varepsilon^{i} \varepsilon^{j} \varepsilon^{k} \varepsilon^{h} \operatorname{det}\left[A^{i} B^{j} C^{k} D^{h}\right]=0
$$

hence

$$
\sum_{0 \leq i, j, k, h \leq m}^{i+j+k+h=m} \operatorname{det}\left[A^{i} B^{j} C^{k} D^{h}\right]=0, \quad(m=0,1,2, \ldots) .
$$

\section{Calculating $\Omega_{1}$}

Corresponding to $m=0$, equation (47) gives

$$
\operatorname{det} S_{0}=0, \quad \Leftrightarrow\left|\begin{array}{cc}
s_{21}^{0} & s_{24}^{0} \\
s_{31}^{0} & s_{21}^{0}
\end{array}\right|\left|\begin{array}{cc}
s_{12}^{0} & s_{13}^{0} \\
s_{42}^{0} & s_{12}^{0}
\end{array}\right|=0 .
$$


From (27) it follows $S_{0}=\int^{1} B_{0}$, thus we have

$$
s_{i j}^{0}=\left\langle b_{i j}\right\rangle, \quad i, j=1,2,3,4 .
$$

where $S_{0}=\left[s_{i j}^{0}\right]$ and $\langle f\rangle=\int^{1} f=\int_{0}^{1} f\left(y_{3}\right) d y_{3}$. From (48) it implies case 1: $\left(s_{21}^{0}\right)^{2}=s_{31}^{0} s_{24}^{0} \Longrightarrow$

$$
\Omega_{1}=\left[\left\langle\tilde{\omega}_{1111}\right\rangle-\left\langle\frac{\tilde{\omega}_{3311}^{2}}{\tilde{\omega}_{3}}\right\rangle+\frac{\left\langle\frac{\tilde{\omega}_{3311}}{\tilde{\omega}_{3}}\right\rangle^{2}}{\left\langle\frac{1}{\tilde{\omega}_{3}}\right\rangle}\right]\langle\tilde{\rho}\rangle^{-1} .
$$

case 2: $\left(s_{12}^{0}\right)^{2}=s_{13}^{0} s_{42}^{0} \Longrightarrow$

$$
\Omega_{1}=\left[\left\langle\tilde{\omega}_{1313}\right\rangle-\left\langle\frac{\tilde{\omega}_{3131}^{2}}{\tilde{\omega}_{1}}\right\rangle+\frac{\left\langle\frac{\tilde{\omega}_{3131}}{\tilde{\omega}_{1}}\right\rangle^{2}}{\left\langle\frac{1}{\tilde{\omega}_{1}}\right\rangle}\right]\langle\tilde{\rho}\rangle^{-1} .
$$

Proving $\Omega_{2}=0$.

From the definition of $S_{i}$, we have

$$
S_{1}=\int^{1} B_{0} \int B_{0}+\int^{1} B_{1}=\left[\begin{array}{cccc}
s_{11}^{1} & 0 & 0 & s_{14}^{1} \\
0 & s_{22}^{1} & s_{23}^{1} & 0 \\
s_{31}^{1} & s_{32}^{1} & s_{33}^{1} & 0 \\
s_{41}^{1} & s_{42}^{1} & 0 & s_{44}^{1}
\end{array}\right]
$$

where

$$
\begin{gathered}
\int^{y_{3}} \Gamma_{1} \int \Gamma_{2}=\int_{0}^{y_{3}} \Gamma_{1}\left(x_{1}\right) d x_{1} \int_{0}^{x_{1}} \Gamma_{2}\left(x_{2}\right) d x_{2} \\
\int^{y_{3}} \Gamma_{1} \int \Gamma_{2} \int \Gamma_{3}=\int_{0}^{y_{3}} \Gamma_{1}\left(x_{1}\right) d x_{1} \int_{0}^{x_{1}} \Gamma_{2}\left(x_{2}\right) d x_{2} \int_{0}^{x_{2}} \Gamma_{2}\left(x_{3}\right) d x_{3}, \ldots, y_{3} \in[0,1] .
\end{gathered}
$$

Corresponding to $m=1$, equation (47) gives

$$
\operatorname{det}\left[A^{1} B^{0} C^{0} D^{0}\right]+\operatorname{det}\left[A^{0} B^{1} C^{0} D^{0}\right]+\operatorname{det}\left[A^{0} B^{0} C^{1} D^{0}\right]+\operatorname{det}\left[A^{0} B^{0} C^{0} D^{1}\right]=0 .
$$

Taking into account (52) and $s_{31}^{1}=s_{42}^{1}=\Omega_{2}\langle\tilde{\rho}\rangle$, equation (53) provides

$$
\Omega_{2}\langle\tilde{\rho}\rangle\left\{s_{24}^{0}\left[\left(s_{12}^{0}\right)^{2}-s_{13}^{0} s_{42}^{0}\right]+s_{13}^{0}\left[\left(s_{21}^{0}\right)^{2}-s_{31}^{0} s_{24}^{0}\right]\right\}=0 .
$$

From (50) and (51): if $\left(s_{12}^{0}\right)^{2}-s_{13}^{0} s_{42}^{0}=0$ then $\left(s_{21}^{0}\right)^{2}-s_{31}^{0} s_{24}^{0} \neq 0$ or, if $\left(s_{12}^{0}\right)^{2}-s_{13}^{0} s_{42}^{0} \neq 0$ then $\left(s_{21}^{0}\right)^{2}-s_{31}^{0} s_{24}^{0}=0$. Hence, we get from (54)

$$
\Omega_{2}=0 \text {. }
$$




\section{Calculating $\Omega_{3}$}

From the definitions of matrices $S_{i}, T_{i}$, we have

$$
S_{2}=T_{2}(1)+\int^{1} B_{2}=\left[\begin{array}{cccc}
0 & s_{12}^{2} & s_{13}^{2} & 0 \\
s_{21}^{2} & 0 & 0 & s_{24}^{2} \\
s_{31}^{2} & 0 & 0 & s_{34}^{2} \\
0 & s_{42}^{2} & s_{43}^{2} & 0
\end{array}\right],
$$

where

$$
T_{2}=\int^{y_{3}} B_{0} \int B_{0} \int B_{0}=\left[\begin{array}{cccc}
0 & t_{12}^{2} & t_{13}^{2} & 0 \\
t_{21}^{2} & 0 & 0 & t_{24}^{2} \\
t_{31}^{2} & 0 & 0 & t_{34}^{2} \\
0 & t_{42}^{2} & t_{43}^{2} & 0
\end{array}\right] .
$$

Corresponding to $m=2$, equation (47) gives

$$
\begin{aligned}
& \operatorname{det}\left[A^{2} B^{0} C^{0} D^{0}\right]+\operatorname{det}\left[A^{0} B^{2} C^{0} D^{0}\right]+\operatorname{det}\left[A^{0} B^{0} C^{2} D^{0}\right]+\operatorname{det}\left[A^{0} B^{0} C^{0} D^{2}\right] \\
& +\operatorname{det}\left[A^{1} B^{1} C^{0} D^{0}\right]+\operatorname{det}\left[A^{1} B^{0} C^{1} D^{0}\right]+\operatorname{det}\left[A^{1} B^{0} C^{0} D^{1}\right] \\
& +\operatorname{det}\left[A^{0} B^{1} C^{1} D^{0}\right]+\operatorname{det}\left[A^{0} B^{1} C^{0} D^{1}\right]+\operatorname{det}\left[A^{0} B^{0} C^{1} D^{1}\right]=0 .
\end{aligned}
$$

We introduce the quantities $M, a, b$ defined as

$$
M=\operatorname{det}\left[A^{1} B^{1} C^{0} D^{0}\right]+\operatorname{det}\left[A^{1} B^{0} C^{1} D^{0}\right]+\operatorname{det}\left[A^{0} B^{1} C^{0} D^{1}\right]+\operatorname{det}\left[A^{0} B^{0} C^{1} D^{1}\right],
$$

and

$$
a=\left|\begin{array}{cc}
s_{12}^{0} & s_{13}^{0} \\
s_{42}^{0} & s_{43}^{0}
\end{array}\right|, \quad b=\left|\begin{array}{cc}
s_{21}^{0} & s_{24}^{0} \\
s_{31}^{0} & s_{34}^{0}
\end{array}\right| .
$$

Taking into account $\Omega_{2}=0$, from (52), (56) and (58) we have

Case 1: $\left(s_{21}^{0}\right)^{2}=s_{31}^{0} s_{24}^{0}$, then $b=0 \Rightarrow \operatorname{det}\left[A_{0} B_{2} C_{0} D_{0}\right]=\operatorname{det}\left[A_{0} B_{0} C_{2} D_{0}\right]=0$, henceforth:

$$
\Omega_{3}=\left(\langle\tilde{\rho}\rangle s_{24}^{0}\right)^{-1}\left[s_{21}^{2} s_{34}^{0}-t_{31}^{2}(1) s_{24}^{0}+s_{34}^{2} s_{21}^{0}-s_{24}^{2} s_{31}^{0}+M a^{-1}\right] .
$$

Case 2: $\left(s_{12}^{0}\right)^{2}=s_{13}^{0} s_{42}^{0}$, then $a=0 \Rightarrow \operatorname{det}\left[A^{2} B^{0} C^{0} D^{0}\right]=\operatorname{det}\left[A^{0} B^{0} C^{0} D^{2}\right]=0$, hence:

$$
\Omega_{3}=\left(\langle\tilde{\rho}\rangle s_{42}^{0}\right)^{-1}\left[s_{12}^{2} s_{43}^{0}-s_{13}^{2} s_{42}^{0}+s_{43}^{2} s_{12}^{0}-t_{42}^{2}(1) s_{13}^{0}+M b^{-1}\right] .
$$




\section{CALCULATING COEFFICIENTS OF HIGHER ORDER}

In this section, we will prove that $\Omega_{2 n}=0$ and construct recurrent formulae for calculating $\Omega_{2 n+1}$. Before doing that, some definitions and lemmas are introduced.

Definition 1: MT1, MT2 are sets of matrices which are of the form:

$$
m t 1=\left[\begin{array}{cccc}
\times & 0 & 0 & \times \\
0 & \times & \times & 0 \\
0 & \times & \times & 0 \\
\times & 0 & 0 & \times
\end{array}\right], \quad m t 2=\left[\begin{array}{cccc}
0 & \times & \times & 0 \\
\times & 0 & 0 & \times \\
\times & 0 & 0 & \times \\
0 & \times & \times & 0
\end{array}\right]
$$

respectively.

Formulas (62) indicate entries being definitely zero of the matrices $m t 1$ and $m t 2$.

From the definition of $M T 1$ and $M T 2$, we immediately have the following lemmas:

Lemma 2: Summation of matrices in MT1 (MT2) belongs to MT1 (MT2).

Lemma 3: Let $m t 1 \in M T 1, m t 2 \in M T 2$, then: $B_{j} m t 2 \in M T 1 ; B_{j} m t 1 \in M T 2$ for all $j \geq 0, j \in \mathbb{N}$.

Lemma 4: The determinant of a matrix which is formed by replacing any column of a $m t 1$-matrix (mt2-matrix) with the corresponding column of a $m t 2$-matrix (mt1-matrix) is equal to zero.

We now prove the following statement:

$$
\Omega_{2 n}=0, \quad \forall n=1,2,3 \ldots
$$

Proof: It has been proved in the previous section that $\Omega_{2}=0$. Thus, the above statement holds true for $n=1$. Assume that statement (63) holds true for any $j=1,2, \ldots, n-1$, that means: $\Omega_{2}=\Omega_{4}=\ldots=\Omega_{2 n-2}=0$. We will prove (63) is true for $j=n$.

From the definition of matrix $B_{k}$, it is seen that $B_{k} \in M T 2, \forall k \geq 0$. The inductive assumption gives

$$
B_{2 j-1}=\tilde{\rho} \Omega_{2 j}=0 \quad \forall j=1,2, \ldots n-1 .
$$

We will show that

$$
R_{2 j-1} \in M T 1, R_{2 j} \in M T 2, \quad \forall j=0,1, \ldots, n-1 .
$$

Indeed, $B_{0} \in M T 2 \Rightarrow R_{0}=\int^{y_{3}} B_{0} \in M T 2$, and $R_{-1}=I \in M T 1$. Hence (65) is true for $j=0$. Assume that (65) is true for every $j=0,1,2, \ldots, k-1$ with $k \leq n-1$, that means:

$$
\left\{\begin{array}{l}
R_{-1}, R_{1}, R_{3}, \ldots R_{2 k-3} \in M T 1, \\
R_{0}, R_{2}, \ldots R_{2 k-2} \in M T 2, \quad k \leq n-1 .
\end{array}\right.
$$

We will prove that (65) is true for $j=k$, that means: 


$$
R_{2 k-1} \in M T 1, R_{2 k} \in M T 2, \quad k \leq n-1 .
$$

Indeed, from (27) and (64) we have (noting that $k \leq n-1$ )

$$
\begin{aligned}
R_{2 k-1} & =\int^{y_{3}} B_{2 k-2} R_{0}+\int^{y_{3}} B_{2 k-4} R_{2}+\ldots+\int^{y_{3}} B_{0} R_{2 k-2} \\
R_{2 k} & =\int^{y_{3}} B_{2 k}+\int^{y_{3}} B_{2 k-2} R_{1}+\ldots+\int^{y_{3}} B_{2} R_{2 k-3}+\int^{y_{3}} B_{0} R_{2 k-1} .
\end{aligned}
$$

From Lemmas $2,3,(66),(68)$ and noting that $B_{k} \in M T 2, \forall k \geq 0$ it deduces (67), i. e. (65) is proved. From (28) and (64) we have

$$
T_{2 n-1}=\int^{y_{3}} B_{2 n-2} R_{0}+\int^{y_{3}} B_{2 n-4} R_{2}+\cdots+\int^{y_{3}} B_{0} R_{2 n-2} .
$$

Therefore, $T_{2 n-1} \in M T 1$ according to Lemmas $2,3,(65)$ and the fact that $B_{k} \in$ $M T 2, \forall k \geq 0$. From (30), we have

$$
R_{2 n-1}=\int^{y_{3}} B_{2 n-1}+T_{2 n-1}
$$

This yields

$$
R_{2 n-1}=\left[\begin{array}{cccc}
t_{11}^{2 n-1} & 0 & 0 & t_{14}^{2 n-1} \\
0 & t_{22}^{2 n-1} & t_{23}^{2 n-1} & 0 \\
\Omega_{2 n} \int^{y_{3}} \tilde{\rho} & t_{32}^{2 n-1} & t_{33}^{2 n-1} & 0 \\
t_{41}^{2 n-1} & \Omega_{2 n} \int^{y_{3}} \tilde{\rho} & 0 & t_{44}^{2 n-1}
\end{array}\right] .
$$

Since $S_{2 n-1}=R_{2 n-1}(1)$, it follows

$$
S_{2 n-1}=\left[\begin{array}{cccc}
t_{11}^{2 n-1}(1) & 0 & 0 & t_{14}^{2 n-1}(1) \\
0 & t_{22}^{2 n-1}(1) & t_{23}^{2 n-1}(1) & 0 \\
\langle\tilde{\rho}\rangle \Omega_{2 n} & t_{32}^{2 n-1}(1) & t_{33}^{2 n-1}(1) & 0 \\
t_{41}^{2 n-1}(1) & \langle\tilde{\rho}\rangle \Omega_{2 n} & 0 & t_{44}^{2 n-1}(1)
\end{array}\right] .
$$

On view of (65) and $S_{n-1}=R_{n-1}(1)$ for every $n \geq 0$, it is clear that

$$
\left\{\begin{array}{l}
S_{-1}, S_{1}, S_{3}, \ldots, S_{2 n-3} \in M T 1 \\
S_{0}, S_{2}, \ldots, S_{2 n-2} \in M T 2 .
\end{array}\right.
$$

With $m=(2 n-1)$, equation (47) is

$$
\begin{aligned}
\operatorname{det}\left[A^{2 n-1} B^{0} C^{0} D^{0}\right]+ & \operatorname{det}\left[A^{0} B^{2 n-1} C^{0} D^{0}\right]+\operatorname{det}\left[A^{0} B^{0} C^{2 n-1} D^{0}\right]+ \\
& +\operatorname{det}\left[A^{0} B^{0} C^{0} D^{2 n-1}\right]+\sum_{0 \leq u, v, s, t<2 n-1}^{u+v+s+t=2 n-1} \operatorname{det}\left[A^{u} B^{v} C^{s} D^{t}\right]=0 .
\end{aligned}
$$


Since $u+v+s+t=2 n-1 \Longrightarrow u+v+s+t$ is an odd number. Therefore, either one of $u, v, s, t$ is odd and the others are even or three of them are odd and the remainder is even. In both cases

$$
\operatorname{det}\left[A^{u} B^{v} C^{s} D^{t}\right]=0, u+v+s+t=2 n-1, \quad 0 \leq u, v, s, t<2 n-1
$$

according to Lemma 4 and taking into account (65). Thus we have

$$
\sum_{0 \leq u, v, s, t<2 n-1}^{u+v+s+t=2 n-1} \operatorname{det}\left[A^{u} B^{v} C^{s} D^{t}\right]=0 .
$$

From (72), it follows

$$
\begin{aligned}
\operatorname{det}\left[A^{2 n-1} B^{0} C^{0} D^{0}\right] & =-s_{24}^{0} s_{31}^{2 n-1}\left[\left(s_{12}^{0}\right)^{2}-s_{13}^{0} s_{42}^{0}\right], \\
\operatorname{det}\left[A^{0} B^{2 n-1} C^{0} D^{0}\right] & =-s_{42}^{2 n-1} s_{13}^{0}\left[\left(s_{21}^{0}\right)^{2}-s_{31}^{0} s_{24}^{0}\right], \\
\operatorname{det}\left[A^{0} B^{0} C^{2 n-1} D^{0}\right] & =0, \operatorname{det}\left[A^{0} B^{0} C^{0} D^{2 n-1}\right]=0 .
\end{aligned}
$$

Substituting (75) and (76) into (74) yields

$$
\Omega_{2 n}\langle\tilde{\rho}\rangle\left\{s_{24}^{0}\left[\left(s_{12}^{0}\right)^{2}-s_{13}^{0} s_{42}^{0}\right]+s_{13}^{0}\left[\left(s_{21}^{0}\right)^{2}-s_{31}^{0} s_{24}^{0}\right]\right\} .
$$

Noting that if $\left(s_{12}^{0}\right)^{2}-s_{13}^{0} s_{42}^{0}=0$ then $\left(s_{21}^{0}\right)^{2}-s_{31}^{0} s_{24}^{0} \neq 0$, or if $\left(s_{12}^{0}\right)^{2}-s_{13}^{0} s_{42}^{0} \neq 0$ then $\left(s_{21}^{0}\right)^{2}-s_{31}^{0} s_{24}^{0}=0$, from $(77)$ it deduces

$$
\Omega_{2 n}=0 \text {. }
$$

The proof of the statement (63) is finished.

Recurrent formulas for $\Omega_{2 n+1}$.

On view of (63) it follows that $B_{2 n-1}=0 \forall n \geq 1$. Therefore, from (28) we have

$$
T_{2 n}=\int^{y_{3}} B_{2 n-2} R_{1}+\int^{y_{3}} B_{2 n-4} R_{3}+\cdots+\int^{y_{3}} B_{0} R_{2 n-1}, n \geq 0 .
$$

Since $B_{2 n-1}=0 \forall n \geq 1$, according to the proof of the statement (65), it is clear that $R_{2 n-1} \in M T 1, R_{2 n} \in M T 2, \forall n \geq 0$. Thus, from (79) it deduces $T_{2 n} \in M T 2(\forall n \geq 0)$ according to Lemmas 2,3 and $B_{k} \in M T 2, \forall k \geq 0$. From (30), we have

$$
R_{2 n}=\int^{y_{3}} B_{2 n}+T_{2 n} .
$$

This and $T_{2 n} \in M T 2$ lead to

$$
R_{2 n}=\left[\begin{array}{cccc}
0 & t_{12}^{2 n} & t_{13}^{2 n} & 0 \\
t_{21}^{2 n} & 0 & 0 & t_{24}^{2 n} \\
t_{31}^{2 n}+\Omega_{2 n+1} \int^{y_{3}} \tilde{\rho} & 0 & 0 & t_{34}^{2 n} \\
0 & t_{42}^{2 n}+\Omega_{2 n+1} \int^{y_{3}} \tilde{\rho} & t_{43}^{2 n} & 0
\end{array}\right] .
$$


Since $S_{2 n}=R_{2 n}(1)$, we have:

$$
S_{2 n}=\left[\begin{array}{cccc}
0 & t_{12}^{2 n}(1) & t_{13}^{2 n}(1) & 0 \\
t_{21}^{2 n}(1) & 0 & 0 & t_{24}^{2 n}(1) \\
t_{31}^{2 n}(1)+\Omega_{2 n+1}\langle\tilde{\rho}\rangle & 0 & 0 & t_{34}^{2 n}(1) \\
0 & t_{42}^{2 n}(1)+\Omega_{2 n+1}\langle\tilde{\rho}\rangle & t_{43}^{2 n}(1) & 0
\end{array}\right]
$$

Corresponding to $m=2 n$, equation (47) is

$$
\begin{aligned}
\operatorname{det}\left[A^{2 n} B^{0} C^{0} D^{0}\right]+\operatorname{det}\left[A^{0} B^{2 n} C^{0} D^{0}\right] & \\
+\operatorname{det}\left[A^{0} B^{0} C^{2 n} D^{0}\right]+\operatorname{det}\left[A^{0} B^{0} C^{0} D^{2 n}\right] & \\
+\sum_{0 \leq u, v, s, t<2 n}^{u+v+s+t=2 n} & \operatorname{det}\left[A^{u} B^{v} C^{s} D^{t}\right]=0 .
\end{aligned}
$$

From (81), we have

$$
\begin{aligned}
\operatorname{det}\left[A^{2 n} B^{0} C^{0} D^{0}\right] & =a\left(s_{21}^{2 n} s_{34}^{0}-s_{31}^{2 n} s_{24}^{0}\right), \\
\operatorname{det}\left[A^{0} B^{2 n} C^{0} D^{0}\right] & =b\left(s_{12}^{2 n} s_{43}^{0}-s_{42}^{2 n} s_{13}^{0}\right), \\
\operatorname{det}\left[A^{0} B^{0} C^{2 n} D^{0}\right] & =b\left(s_{12}^{0} s_{43}^{2 n}-s_{42}^{0} s_{13}^{2 n}\right), \\
\operatorname{det}\left[A^{0} B^{0} C^{0} D^{2 n}\right] & =a\left(s_{21}^{0} s_{34}^{2 n}-s_{31}^{0} s_{24}^{2 n}\right) .
\end{aligned}
$$

It follows from (82) that

Case 1: $\left(s_{21}^{0}\right)^{2}=s_{31}^{0} s_{24}^{0} \Rightarrow b=0 \Rightarrow \operatorname{det}\left[A^{0} B^{2 n} C^{0} D^{0}\right]=\operatorname{det}\left[A^{0} B^{0} C^{2 n} D^{0}\right]=0$, then

$$
s_{21}^{2 n} s_{34}^{0}-s_{31}^{2 n} s_{24}^{0}+s_{34}^{2 n} s_{21}^{0}-s_{24}^{2 n} s_{31}^{0}+\sum_{0 \leq u, v, s, t<2 n}^{u+v+s+t=2 n} \operatorname{det}\left[A^{u} B^{v} C^{s} D^{t}\right] a^{-1}=0 .
$$

This provides

$$
\Omega_{2 n+1}=\left(\langle\tilde{\rho}\rangle s_{24}^{0}\right)^{-1}\left[\begin{array}{c}
s_{21}^{2 n} s_{34}^{0}-t_{31}^{2 n}(1) s_{24}^{0}+s_{34}^{2 n} s_{21}^{0}-s_{24}^{2 n} s_{31}^{0}+ \\
+\sum_{0 \leq u, v, s, t<2 n}^{u+v+s+t=2 n} \operatorname{det}\left[A^{u} B^{v} C^{s} D^{t}\right] a^{-1}
\end{array}\right],
$$

for every $n \geq 1$.

Case 2: $\left(s_{12}^{0}\right)^{2}=s_{13}^{0} s_{42}^{0} \Rightarrow a=0 \Rightarrow \operatorname{det}\left[A^{2 n} B^{0} C^{0} D^{0}\right]=\operatorname{det}\left[A^{0} B^{0} C^{0} D^{2 n}\right]=0$, then

$$
s_{12}^{2 n} s_{43}^{0}-s_{13}^{2 n} s_{42}^{0}+s_{43}^{2 n} s_{12}^{0}-s_{42}^{2 n} s_{13}^{0}+\sum_{0 \leq u, v, s, t<2 n}^{u+v+s+t=2 n} \operatorname{det}\left[A^{u} B^{v} C^{s} D^{t}\right] b^{-1}=0 .
$$


This yields

$$
\Omega_{2 n+1}=\left(\langle\tilde{\rho}\rangle s_{42}^{0}\right)^{-1}\left[\begin{array}{c}
s_{12}^{2 n} s_{43}^{0}-s_{13}^{2 n} s_{42}^{0}+s_{43}^{2 n} s_{12}^{0}-t_{42}^{2}(1) s_{13}^{0}+ \\
+\sum_{0 \leq u, v, s, t<2 n}^{u+v+s+t=2 n} \operatorname{det}\left[A^{u} B^{v} C^{s} D^{t}\right] b^{-1}
\end{array}\right],
$$

for every $n \geq 1$. The formulas (83) and (84) are the recurrent formulas for determining $\Omega_{2 n+1}, n \geq 1$.

\section{CONCLUSIONS}

In this paper, we consider the long-Lamb wave propagation in a periodically layered, compressible elastic medium subject to a homogeneous initial deformation. Taking the assumption $\varepsilon=k . h<<1$, the dispersion of the wave is expressed as a power series of the small parameter $\varepsilon$ whose coefficients are $\Omega_{m}, m \geq 1$. We have derived the expressions for $\Omega_{1}, \Omega_{3}$, and proved that $\Omega_{2}=0$. We have also established the recurrent formulae for calculating the non-zero higher-order coefficients $\Omega_{2 k+1}, k \geq 2$, and proved that $\Omega_{2 k}=$ $0 \forall k \geq 1$. Based on these formulae, the solution with any order of accuracy can be obtained.

\section{REFERENCES}

[1] J. D. Achenbach, Wave propagation in Elastic Solids, North-Holland, Amsterdam 1973.

[2] L. M. Brekhovskikh and V. Goncharov, Mechanics of continua and wave dynamics, SpringerVerlag, New York, 1994.

[3] H. F. Tiersten, Elastic surface waves guided by thin films, J. Appl. Phys. 40 (1969) 770-789.

[4] G. A. Hergemier and A. H. Nayfeh, A continuum theory for wave propagation in laminated composite, J. Appl. Mech. 40 (1973) 503-510.

[5] A. H. Nayfeh, Time-harmonic waves propagating normal to the layers of multilayered periodic media, J. Appl. Mech. 41 (1974) 92-96.

[6] A. Norris and F. Santosa, Shear wave propagation in a periodically layered medium - an asymptotic theory, Wave Motion 16 (1992) 33-55

[7] Pham Chi Vinh, Propagation of the floquet wave along to layers of multilayered periodic media with homogenenous initial deformations, Proc. NSCT of Vietnam 6 (2) (1994) 21-31.

[8] A. V. Pichugin and G. A. Rogerson, An asymptotic membrane-like theory for a long-wave motion in a pre-stressed elastic layer, Proc. R. Soc. Lond. A 458 (2002) 1447-1468.

[9] E. V. - Ide, G. A. Rogerson, Long wave asymptotic integration of the governing equations for a pre-st: issed incompressible elastic layer with fixed faces, Wave Motion 36 (2002) 287-304.

[10] A. L. Shuvalov, A. G. Every, Some properties of surface acoustic waves in anisotropic-coated solids, studied by the impedance method, Wave Motion 36 (2002) 257-273.

[11] A. L. Shuvalov, O. Poncelet, M. Deschamps, C. Baron, Long-wavelength dispersion of acoustic waves in transversely inhomogeneous anisotropic plates, Wave Motion 42 (2005) 367-382.

[12] L. A. Prikazchikova, I. A. Alejnikov, G. A. Rogerson, Pade approximations for low-frequency motion in a pre-stressed compressible elastic layer, Mech. Comm. Res. 33 (2006) 699-674. 
[13] L. Yu. Kossovich, R. R. Moukhomodiarov, R. A. Rogerson, Symmetric low-frequency motion in incompressible transversely isotropic elastic plates, Mech. Comm. Res. 33 (2006) 460-469.

[14] J. Kaplunov, E. Nolde and G. A. Rogerson, An asymptotic analysis of initial-value problems for thin elastic plates, Proc. R. Soc. Lond. A 462 (2006) 2541-2561.

[15] O. Poncelet, A. L. Suvalov, J. Kaplunov, Approximation of the flexural velocity branch in plates, Int. J. Solids Struct. 43 (2006), 6329-6346.

[16] J. D. Steighmann and R. W. Ogden, Surface waves supported by thin-film/substrate interactions, IMA J. Appl. Math. 72 (2007) 730-747.

[17] A. L. Shuvalov, A. G. Every, On the long-wave onset of dispersion of the surface-wave velocity in coated solids, Wave Motion 45 (2008) 857-863.

[18] Guz A. N., Elastic waves in the body with initial stresses, I. General theory, Naukova Dumka, Kiev 1986. (in Russian)

[19] Pham Chi Vinh, Some problems of wave propagation in pre-stressed media (in Vietnamese), PhD dissertation, Hanoi 1986.

Received May 12, 2009

\section{KHAI TRIỂN TIỆ CẬN CỦA PHƯƠNG TRÌNH TÁN SẮC CỦA SÓNG LAMB TRONG CÁC MÔI TRƯờNG ĐÀN HỒI PHÂN LỚP TUẦN HOÀN}

Bài báo nghiên cứu sự truyền của sóng Lamb trong các môi trường đàn hồi nén được, phân lớp tuần hoàn, có biến dạng trước, trong trường hợp xấp xỉ sóng dài (tức là khi $0<\varepsilon=k \cdot h<<1$, trong đó $k$ là số sóng, $h$ là độ dầy của một chu kỳ). Với giả thiết $\varepsilon<<1$ phương trình tán sắc của sóng được viết dưới dạng:

$$
\frac{\varpi^{2}}{k^{2}}=\Omega_{1}+\varepsilon \Omega_{2}+\varepsilon^{2} \Omega_{3}+\ldots=\sum_{m=0}^{\infty} \varepsilon^{m} \Omega_{m+1}
$$

Mục đích chính của bài báo là tìm các công thức xác định các hệ số $\Omega_{i}(i \geq 1) \ldots$ Các tác giả đã chứng minh được rằng $\Omega_{2 n}=0$ với mọi $n \geq 1$, tìm ra các công thức xác định $\Omega_{1}, \Omega_{3}$, và xây dựng được các công thức truy hồi để tính $\Omega_{2 n+1}, n \geq 2$. Sử dụng các công thức này, ta có thể tìm được nghiệm với độ chính xác tùy ý. Bài báo là sự mở rộng của nghiên cứu thực hiện bởi Noris và Santosa [Norris A. and Santosa F., Wave Motion 16 (1992), 33-55] từ sóng SH (sóng một thành phần) sang sóng Lamb (sóng hai thành phần). 